\title{
First $\mathrm{NH}_{3}$ detection of the Orion Bar ${ }^{\star}$
}

\author{
B. Larsson ${ }^{1}$, R. Liseau ${ }^{1}$, P. Bergman ${ }^{2}$, P. Bernath ${ }^{3}$, J. H. Black ${ }^{2}$, R. S. Booth ${ }^{2}$, V. Buat ${ }^{4}$, C. L. Curry ${ }^{3}$, P. Encrenaz ${ }^{5}$,

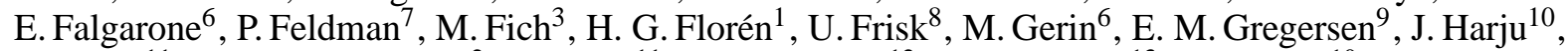 \\ T. Hasegawa ${ }^{11}$, L. E. B. Johansson ${ }^{2}$, S. Kwok ${ }^{11}$, A. Lecacheux ${ }^{12}$, T. Liljeström ${ }^{13}$, K. Mattila ${ }^{10}$, G. F. Mitchell ${ }^{14}$, \\ L. H. Nordh ${ }^{15}$, M. Olberg ${ }^{2}$, G. Olofsson ${ }^{1}$, L. Pagani ${ }^{5}$, R. Plume ${ }^{11}$, I. Ristorcelli ${ }^{16}$, Aa. Sandqvist ${ }^{1}$, F. v. Schéele ${ }^{8}$, \\ N. F. H. Tothill ${ }^{14}$, K. Volk ${ }^{9}$, C. D. Wilson ${ }^{9}$, and Å. Hjalmarson ${ }^{2}$
}

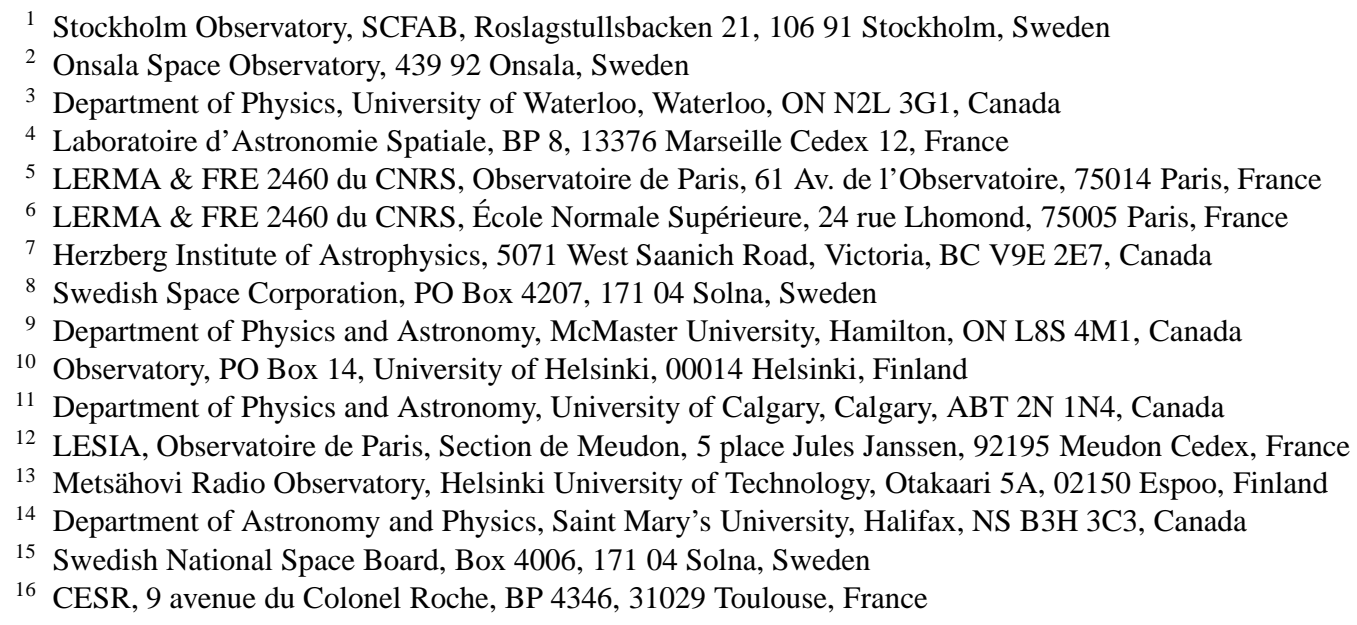

Received 29 November 2002 / Accepted 1 February 2003

\begin{abstract}
Odin has successfully observed three regions in the Orion A cloud, i.e. Ori KL, Ori S and the Orion Bar, in the $572.5 \mathrm{GHz}$ rotational ground state line of ammonia, ortho- $\mathrm{NH}_{3}(J, K)=(1,0) \rightarrow(0,0)$, and the result for the Orion Bar represents the first detection in an ammonia line. Several velocity components are present in the data. Specifically, the observed line profile from the Orion Bar can be decomposed into two components, which are in agreement with observations in high- $J$ CO lines by Wilson et al. (2001). Using the source model for the Orion Bar by these authors, our Odin observation implies a total ammonia abundance of $\mathrm{NH}_{3} / \mathrm{H}_{2}=5 \times 10^{-9}$.
\end{abstract}

Key words. ISM: individual objects: Orion A - clouds - molecules - abundances - stars: formation

\section{Introduction}

The central parts of the Orion A cloud contain several different source regions, among which one can identify Orion KL, Orion S and the Orion Bar (see, e.g., Fig. 3 of Wilson et al. 2001). On angular scales of two arcminutes (the Odin beam size, Sect. 2), the Ori KL region, in particular, harbours a highly complex source structure, apparently with several components along the line of sight. The region has been reviewed by Genzel $\&$ Stutski (1989) and more recent results and references can be

Send offprint requests to: B. Larsson, e-mail: bem@astro.su.se

* Based on observations with Odin, a Swedish-led satellite project funded jointly by the Swedish National Space Board (SNSB), the Canadian Space Agency (CSA), the National Technology Agency of Finland (Tekes) and Centre National d'Études Spatiales (CNES). The Swedish Space Corporation has been the industrial prime contractor. found in the papers of Wilson et al. (2001) and of Wiseman \& Ho (1998). The latter authors presented VLA-maps in two inversion lines of para-ammonia, showing the emission to arise from finger-like structures. However, as in previous observations (e.g. Ho et al. 1979), the Orion Bar was not detected.

The structure of the $\mathrm{NH}_{3}$ molecule and its versatility as an astrophysical tool have been discussed by Ho \& Townes (1983). These authors also provide an energy level diagram. The rotational ground state line of ammonia, $\mathrm{NH}_{3}(J, K)=$ $(1,0) \rightarrow(0,0)$, has a wavelength of $524 \mu \mathrm{m}$ and an upper state energy of $28 \mathrm{~K}$ above ground. For the $(1,1)$ and $(2,2)$ inversion lines at $1.3 \mathrm{~cm}$, the corresponding values are $24 \mathrm{~K}$ and $65 \mathrm{~K}$, respectively. The radiative lifetime of the $(1,0) \rightarrow(0,0)$ transition is shorter by orders of magnitude than those of the inversion lines and the line profile could therefore potentially 
be probing regions of very different excitation conditions (cf. Table 2 of Liseau et al., this issue). In particular for dynamical studies, the $\mathrm{NH}_{3}$ line is expected to complement or, in regions of undetectably low water vapour abundance, to substitute for the ground state line of ortho- $\mathrm{H}_{2} \mathrm{O}\left(1_{10}-1_{01}\right)$, since these resonance lines share the property of being "effectively transparent" up to very high optical depths, as they exhibit an essentially linear growth of the intensity (for $\tau$ of several hundred; see, e.g., Snell et al. 2000 and Liseau 2001).

Using the Kuiper Airborne Observatory (KAO), Keene et al. (1983) observed the $\mathrm{NH}_{3}(1,0) \rightarrow(0,0)$ line toward Ori KL and made a five point cross with $1^{\prime}$ spacing. Their observations will be compared to those presented in this letter, which have been obtained with Odin (Sect. 2), a spaceborne submillimeter telescope (Frisk et al., Hjalmarson et al. and Nordh et al., this issue). The reductions of these data, which required some special and careful treatment, are described in detail in Sect. 3 and the results are presented in Sect. 4. The concluding discussion (Sect. 5) of this Letter will focus on the $\mathrm{NH}_{3}$ emission from the Orion Bar, as this provides an entirely new piece of information for the complex Orion A region.

\section{The Odin observations}

The $\mathrm{NH}_{3}$ observations of Orion $\mathrm{A}$ were made on September 27 to 29, 2001, simultaneously with observations of the $\mathrm{H}_{2}^{18} \mathrm{O}\left(1_{10}-1_{01}\right)$ and $\mathrm{O}_{2}\left(1_{1}-1_{0}\right)$ lines (Olofsson et al. and Pagani et al., this issue). Aiming at Ori KL, the coordinates of the $(0,0)$ position were $R A=5^{\mathrm{h}} 35^{\mathrm{m}} 15 \mathrm{~s} 1$ and Dec $=-5^{\circ} 22^{\prime} 12^{\prime \prime}(\mathrm{J} 2000)$. The absolute pointing of the Odin telescope is presently known to within $30^{\prime \prime}$. At $572.5 \mathrm{GHz}$ the half power beam width is $2^{\prime}$. In addition, two positions with the nominal relative offsets in $\operatorname{arcmin}(0,-2$; Ori $S)$ and $(+2,-2$; Orion Bar) were observed. During the observations, the relative pointing accuracy $(\mathrm{rms})$ was $(\Delta \mathrm{RA}, \Delta \mathrm{Dec}) \lesssim\left(7^{\prime \prime}, 14^{\prime \prime}\right)$ The data were obtained in a sky-switching mode (Frisk et al. and Hjalmarson et al., this issue) by observing a total of $4.5 \mathrm{hr}$ each on-source and on blank sky, with $10 \mathrm{~s}$ integrations per individual scan. As back-end, a digital hybrid autocorrelator (AC) was used, with an $800 \mathrm{MHz}$ bandwidth at the resolution of $1 \mathrm{MHz}\left(0.5 \mathrm{~km} \mathrm{~s}^{-1}\right)$.

\section{Data reductions}

The phase-lock system for the $572 \mathrm{GHz}$ receiver is not working properly. However, it is reasonably well locked at the tuning frequency and the telluric ozone line $\mathrm{O}_{3}\left(J_{K^{-}, K^{+}}=30_{4,26} \rightarrow\right.$ $\left.30_{3,27}\right) 572.9 \mathrm{GHz}$ is frequently observed along parts of the Odin orbit, drifting across the receiver band. The line is sufficiently close in frequency to the ammonia line $\mathrm{NH}_{3}(J, K)=$ $(1,0) \rightarrow(0,0) 572.5 \mathrm{GHz}$ that it can be used to establish the observing frequency scale, and hence allows us to restore the $\mathrm{NH}_{3}$ data.

The drift of the $\mathrm{O}_{3}$ line can be expected to correlate with the equipment temperature and, in particular, inversely with the temperature measured at the local oscillator (LO). That this is indeed the case is demonstrated in the upper panel of
Table 1. Gaussian fit parameters for the observed line profiles.

\begin{tabular}{|c|c|c|c|c|c|c|}
\hline \multirow[b]{2}{*}{$\begin{array}{c}\text { Offset } \\
(\operatorname{arcmin})\end{array}$} & \multicolumn{3}{|c|}{ Component 1} & \multicolumn{3}{|c|}{ Component 2} \\
\hline & $\begin{array}{l}T_{0,1} \\
(\mathrm{~K})\end{array}$ & $\begin{array}{c}v_{\mathrm{LSR}, 1} \\
\left(\mathrm{~km} \mathrm{~s}^{-1}\right)\end{array}$ & $\begin{array}{c}\Delta v_{1} \\
\left(\mathrm{~km} \mathrm{~s}^{-1}\right)\end{array}$ & $\begin{array}{l}T_{0,2} \\
(\mathrm{~K})\end{array}$ & $\begin{array}{c}v_{\mathrm{LSR}, 2} \\
\left(\mathrm{~km} \mathrm{~s}^{-1}\right)\end{array}$ & $\begin{array}{c}\Delta v_{2} \\
\left(\mathrm{~km} \mathrm{~s}^{-1}\right)\end{array}$ \\
\hline$(0,0)$ & 2.0 & 9.1 & 4.9 & 0.9 & 9.9 & 16.0 \\
\hline$(0,-2)$ & 1.5 & 8.3 & 5.5 & $\ldots$ & $\ldots$ & $\ldots$ \\
\hline$(+2,-2)$ & 0.35 & 8.1 & 5.4 & 0.35 & 10.5 & 3.3 \\
\hline
\end{tabular}

Fig. 1, where the center channel for the $\mathrm{O}_{3}$ line, $C_{\mathrm{O}_{3}}$ and identified by plus-signs, is shown as a function of the time, expressed as satellite revolution numbers. The solid curve in that panel depicts the relation for the reciprocal LO-temperature, $f\left(T_{\mathrm{LO}}\right) \propto 1 / T_{\mathrm{LO}}$. Subtraction of the $T_{\mathrm{LO}}$ relation from the $\mathrm{O}_{3}$ channel data points results in the swarm of plus signs in the middle panel of Fig. 1. A polynominal fit to these points is shown by the full drawn curve $[=g(\Delta)]$, depicting the temperature behaviour measured at the telescope (main reflector). In the lower panel, the residual displays the final scatter about the fixed central channel for the $\mathrm{O}_{3}$ line ( $\mathrm{rms}=0.7$ channels $=0.36 \mathrm{~km} \mathrm{~s}^{-1}$ ).

The drift corrections to the Orion observations were done by applying the empirically determined relation

$C_{i}=C_{i 0}-\left[f\left(T_{\mathrm{LO}}\right)+g(\Delta)+C_{\mathrm{O}_{3}}\right]$,

where the frequencies are expressed in channel units and where the constant, $C_{\mathrm{O}_{3}}$, is derived from the ozone line observations. The final spectra are given in the main beam temperature scale, defined as

$T_{\mathrm{mb}}=\frac{T_{\mathrm{A}}}{\eta_{\mathrm{mb}}}=\frac{1}{\eta_{\mathrm{mb}}} \frac{T_{\mathrm{on}}-T_{\text {sky }}}{T_{\text {sky }}} T_{\text {sys }}$,

with obvious notations (average $T_{\mathrm{sys}}=3750 \mathrm{~K}$ ). In the upper panel of Fig. 2, the averaged spectral scans ( $T_{\mathrm{A}}$ vs. $C$ ) toward the three observed positions in Orion are shown, together with polynomial fits to their baselines. Finally, the baseline subtracted spectra ( $T_{\mathrm{mb}}$ vs. $\left.v_{\mathrm{LSR}}\right)$ are shown in the lower panel. The choice of polynomials is conservative, as a sinosoidal baseline fit to the remaining standing wave pattern would result in more extended wings in especially the $(0,0)$ spectrum.

\section{Results}

The $\mathrm{NH}_{3}(J, K)=(1,0) \rightarrow(0,0)$ spectra observed toward the three positions in Ori A are shown in Fig. 2 (lower panel). The agreement with the KAO data toward Ori KL, also shown in that figure for comparison, is striking, although the signal-tonoise ratio of the Odin data is far better. Keene et al. (1983) speculated whether there existed some low intensity broad component in their data. That this is indeed the case has now been confirmed by Odin. In our observations, the line intensity peaks at the KL position and decreases by about a factor of two at Ori S and by a factor of five toward the Orion Bar. Changes in radial velocity of the line centers are also evident. In the figure, the results of Gaussian fits to the line profiles are also 

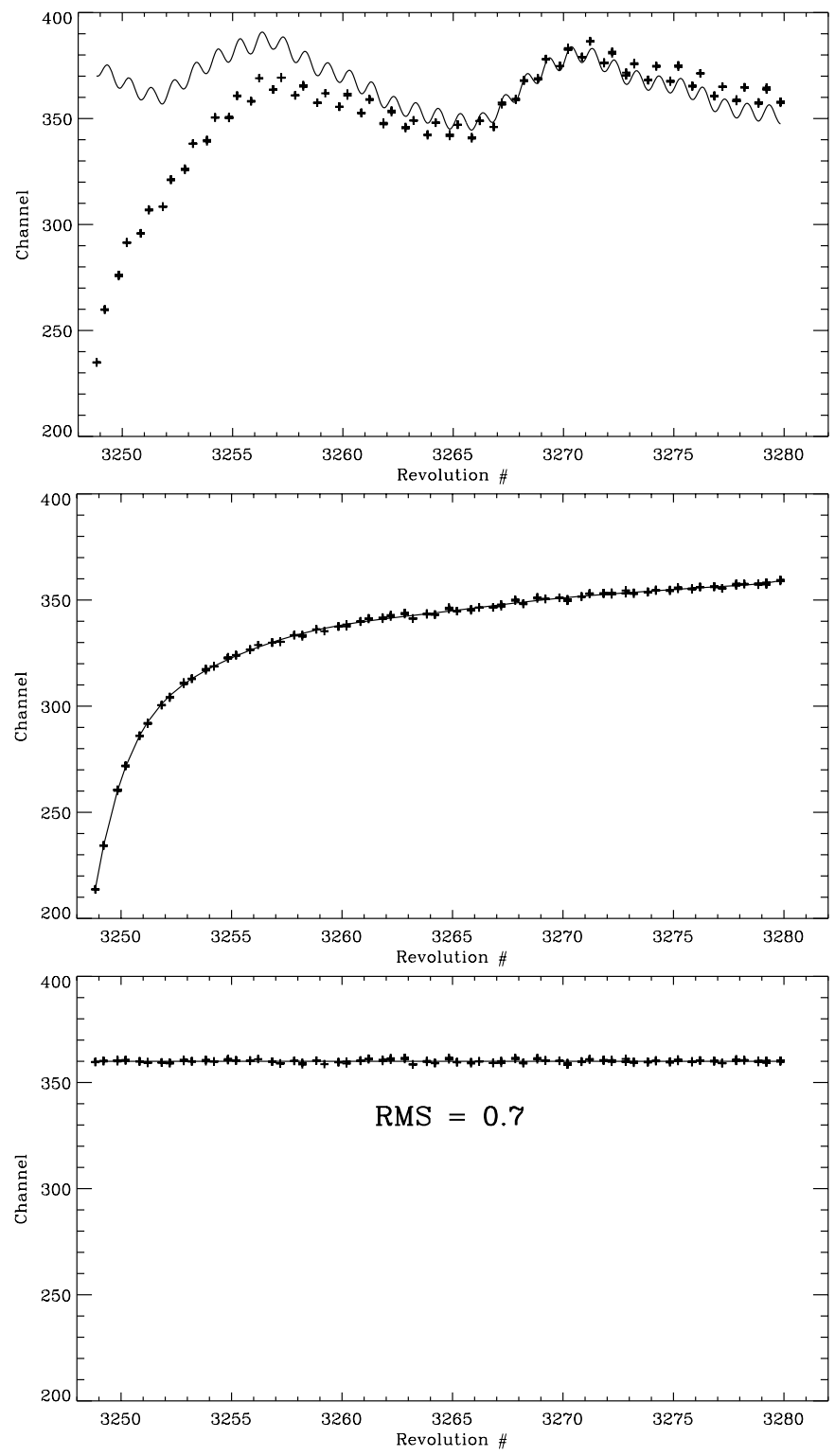

Fig. 1. Correction for frequency drift during the observation. Upper: the plus-signs denote the channel number of the center of the telluric $\mathrm{O}_{3}$ line during the Orion observations, expressed as Odin revolutions. The upper curve (solid line) depicts the behaviour of the reciprocal LO-temperature. Middle: the plus-signs designate the difference between the values shown above. The full-drawn curve is a polynomial fit to these points. This curve describes the average behaviour of the temperature measured at the main reflector. Lower: the rms-spread of the residuals to the polynomial fit corresponds to 0.7 of the AC-channel width $\left(=0.36 \mathrm{~km} \mathrm{~s}^{-1}\right)$.

shown and their parameters are reported in Table 1. Four distinct velocity systems can be identified: (1) a $v_{\mathrm{LSR}}=9 \mathrm{~km} \mathrm{~s}^{-1}$ component of width $5 \mathrm{~km} \mathrm{~s}^{-1}(F W H M)$ is present toward Ori KL, (2) a very broad feature $\left(\Delta v=16 \mathrm{~km} \mathrm{~s}^{-1}\right)$, but centered at $v_{\mathrm{LSR}}=10 \mathrm{~km} \mathrm{~s}^{-1}$, is also present in Ori KL, (3) a blueshifted line $\left(v_{\mathrm{LSR}}=8 \mathrm{~km} \mathrm{~s}^{-1}\right)$ of width $5.5 \mathrm{~km} \mathrm{~s}^{-1}$ toward the outflow source Ori S and the Orion Bar and (4) a component at $v_{\mathrm{LSR}}=10.5 \mathrm{~km} \mathrm{~s}^{-1}$ of width $3.3 \mathrm{~km} \mathrm{~s}^{-1}$ from the Orion Bar, identical to $\mathrm{CO}(J=4-3)$ (Wilson et al. 2001). The result
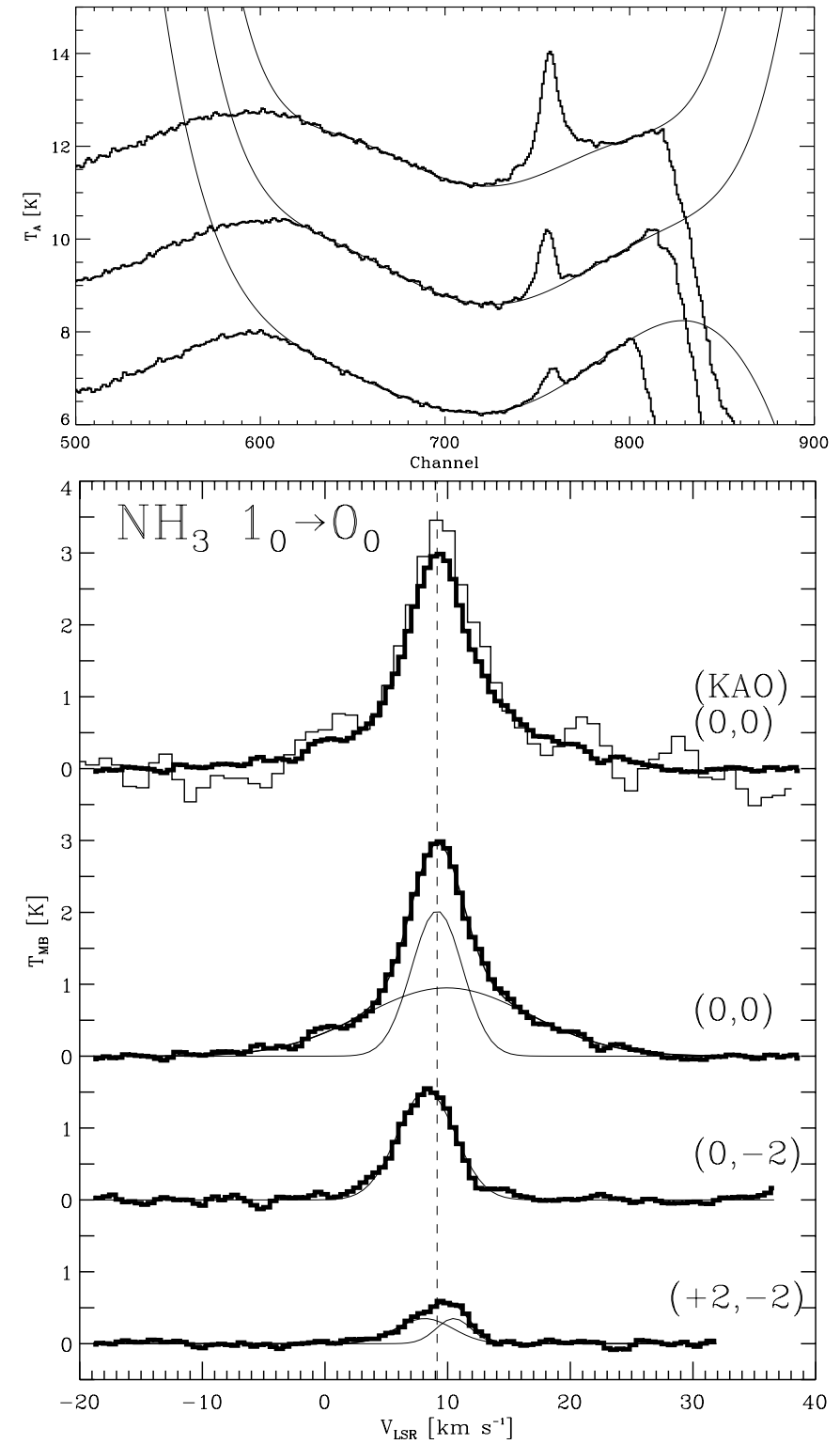

Fig. 2. $\mathrm{NH}_{3}(J, K)=(1,0) \rightarrow(0,0)$ spectra toward Orion A. Upper: the reduced spectra for the three positions discussed in the text (antenna temperature vs. correlator channel) are shown together with the adopted polynomial fits to their baselines. Lower: same as above, but with the baselines subtracted ( $T_{\mathrm{mb}}$ vs. $\left.v_{\mathrm{LSR}}\right)$. The data are displayed as histograms. Uppermost, the KAO observation ( $\left.2^{\prime} F W H M\right)$ by Keene et al. (1983) (thin line) is shown together with the Odin spectrum toward $(0,0)$. The much higher $\mathrm{S} / \mathrm{N}$ of the Odin data aside, the agreement between these two observations is strikingly good. Following below, the spectra toward the three positions observed with Odin are displayed, together with Gaussian fits to the line profiles as thin lines (cf. Table 1). For reference, a vertical dashed line is drawn at $v_{\mathrm{LSR}}=9.1 \mathrm{~km} \mathrm{~s}^{-1}$.

for the Orion Bar represents the first detection in an ammonia line.

The radial velocities found in the $\mathrm{NH}_{3}(1,0) \rightarrow(0,0)$ spectra are different from those quoted for various inversion lines toward Ori KL by Hermsen et al. (1988a), but are in good agreement with those observed toward all positions in the ortho- $\mathrm{H}_{2} \mathrm{O}\left(1_{10}-1_{01}\right) 557 \mathrm{GHz}$ line during an independent 
observing run (Olofsson et al., this issue). The line shapes of these transitions do agree in the direction of Ori $S$ and the Bar, but at the resolution of $1 \mathrm{MHz}$, the $\mathrm{NH}_{3} 572.5 \mathrm{GHz}$ line core toward Ori KL is highly symmetric and does not display any self-absorption like feature as does the $\mathrm{H}_{2} \mathrm{O} 557 \mathrm{GHz}$ line.

\section{Discussion and conclusions}

For the Orion Bar, no previous $\mathrm{NH}_{3}$ line information, other than upper limit results, is in existence. For the interpretation of this observation, we will have to rely on source models based primarily on other data. The observed width of the Bar ranges from about $10^{\prime \prime}$ to $60^{\prime \prime}$ for a variety of molecules (e.g., Tielens et al. 1993; Wilson et al. 2001). Based on observations in high- $J$ CO lines, estimates of the physical parameters of the Orion Bar have been given by Wilson et al. (2001). These authors argue that the geometry is that of a $30^{\prime \prime}$ wide rod in the plane of the sky and we assume that their model is applicable also to $\mathrm{NH}_{3}$. The observed width of the $\mathrm{NH}_{3}$ and $\mathrm{CO}$ lines, $\Delta v=3.3 \mathrm{~km} \mathrm{~s}^{-1}$, can be interpreted as a velocity gradient of $45 \mathrm{~km} \mathrm{~s}^{-1} \mathrm{pc}^{-1}$. The hyperfine structure of the $\mathrm{NH}_{3}$ transition (Townes \& Schawlow 1955; cf. Liseau et al., this issue) does not significantly contribute to the observed width of the line. The column density of $\mathrm{H}_{2}$ is given by Wilson et al. (2001) as $N\left(\mathrm{H}_{2}\right)=2.2 \times 10^{22} \mathrm{~cm}^{-2}$. As kinetic gas temperature we adopt the peak value of their observed CO (7-6) line, $T_{\mathrm{k}}=145 \mathrm{~K}$.

We use a large velocity gradient code to estimate the statistical equilibrium level populations and to solve the equation of radiative transfer along the line of sight. We have calculated the level energies and Einstein- $A$ values according to Poynter \& Kakar (1975), using the dipole moment of Cohen \& Poynter (1974). For the computation of the collision rates, we adopted the values provided by Danby et al. (1988) for collisions with para- $\mathrm{H}_{2}(J=0)$, in conjunction with the assumption of detailed balance for the inverse rates.

For the source model of Wilson et al. (2001), we find the abundance of ortho-ammonia relative to $\mathrm{H}_{2}$ in the Orion Bar to be $X_{\mathrm{o}}\left(\mathrm{NH}_{3}\right)=2.5 \times 10^{-9}$, where the $\mathrm{NH}_{3}(1,0) \rightarrow(0,0)$ line radiation temperature is $T_{\mathrm{R}}=1.7 \mathrm{~K}$, corrected for partial beam filling $(\sim 0.2)$. This result for $X_{\mathrm{o}}$ remains unaltered, if the kinetic temperature were higher, e.g., $T_{\mathrm{k}}=200 \mathrm{~K}$. The model is also in agreement with the upper limits on the inversion lines of Ho et al. (1979) and Wiseman \& Ho (1998), provided the ammonia ortho-to-para ratio is not largely different from unity. This would be expected for a gas at such elevated temperatures, since for ammonia the ortho-to-para ratio $\rightarrow g_{\mathrm{o}} / 2 g_{\mathrm{p}}=1$ as $T_{\mathrm{k}} \rightarrow \infty$. The high temperature ortho-to-para ratio of 1.0 has previously been determined for Ori KL by Morris et al. (1973). For the Orion Bar, such ratio would thus imply a total ammonia abundance $X\left(\mathrm{NH}_{3}\right)=5 \times 10^{-9}$. The ammonia emission region is probably not much narrower than $10^{\prime \prime}$ and average densities do probably not exceed $10^{6} \mathrm{~cm}^{-3}$ by large, so that it is likely that $X\left(\mathrm{NH}_{3}\right)<2 \times 10^{-8}$. These estimates can be compared to the values deduced for the Ori KL components "hot core" $\left(10^{-7}-10^{-6}\right.$, Dishoeck 1993; Hermsen et al. 1988b) and "plateau" $\left(<10^{-8}\right.$, van Dishoeck et al. 1993). The density of the applied model is lower than the critical density of the $(1,0) \rightarrow(0,0)$ transition by more than one order of magnitude and, hence, the line is very subthermally excited $\left(T_{\mathrm{ex}}=11 \mathrm{~K}\right)$. For the inferred abundance, the line is moderately optically thick $(\tau=1)$.

The validity of the presented results rests on the assumption that the CO model by Wilson et al. (2001) is applicable to $\mathrm{NH}_{3}$. The observation of the Orion Bar in the $(3,3)$ inversion line could provide a test, as this line is predicted to be the strongest $\mathrm{NH}_{3}$ transition.

\section{References}

Cohen, E. A., \& Poynter, R. L. 1974, J. Mol. Spec., 53, 131

Danby, G., Flower, D. R., Valiron, P., Schilke, P., \& Walmsley, C. M. 1988, MNRAS, 235, 229

Genzel, R., \& Stutski, J. 1989, ARA\&A, 27, 41

Hermsen, W., Wilson, T. L., Walmsley, C. M., \& Henkel, C. 1988a, A\&A, 201, 285

Hermsen, W., Wilson, T. L., \& Bieging, J. H. 1988b, A\&A, 201, 276

Ho, P. T. P., Barrett, A. H., Myers, P. C., et al. 1979, ApJ, 234, 912

Ho, P. T. P., \& Townes, C. H. 1983, ARA\&A, 21, 239

Keene, J., Blake, G. A., \& Phillips, T. G. 1983, ApJ, 271, L 27

Liseau, R. 2001, in The Promise of the Herschel Space Observatory, ed. G. L. Pilbratt, J. Cernicharo, A. M. Heras, T. Prusti, \& R. Harris, ESA SP-460, 313

Lovas, F. J. 1992, J. Phys. Chem. Ref. Data, 21, 181

Morris, M., Zuckerman, B., Palmer, P., \& Turner, B. E. 1973, ApJ, 186,501

Poynter, R. L., \& Kakar, R. K. 1975, ApJS, 29, 87

Snell, R. L., Howe, J. E., Ashby, M. L. N., et al. 2000, ApJ, 539, L 93

Tielens, A. G. G. M., Meixner, M. M., van der Werf, P. P., et al. 1993, Science, 262, 86

Townes, C. H., \& Schawlow, A. L. 1955, Microwave Spectroscopy (Dover Publications, Inc.)

Wilson, T. L., Muders, D., Kramer, C., \& Henkel, C. 2001, ApJ, 557, 240

Wiseman, J. J., \& Ho, P. T. P. 1998, ApJ, 502, 676

van Dishoeck, E. F., Blake, G. A., Draine, B. T., \& Lunine, J. I. 1993, in Protostars and Planets III, ed. E. H. Levy, \& J. I. Lunine, (University of Arizona Press), 163 